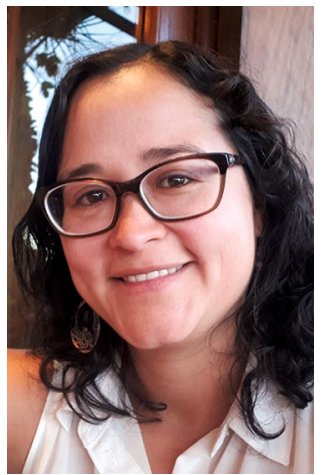

4 Leading a lab is not for the faint hearted; I received my first substantial grant almost 3 years in

Unidad de Genómica Avanzada, Langebio, Centro de Investigación y Estudios Avanzados del Instituto Politécnico Nacional, Irapuato, Guanajuato, Mexico.

e-mail: selene.fernandez@ cinvestav.mx

Check for updates

\title{
Voices of the new generation: being a junior PI in a developing country
}

Selene L. Fernandez-Valverde (1)

Starting a research group in a developing country can be economically, intellectually and personally challenging, but funding and other opportunities may be broader than they may seem from afar.

Almost 5 years ago, I decided to move my whole life across the Pacific Ocean to lead my own research group in my native Mexico. I had spent 8 years in Australia, where I originally intended to continue my academic career, but opportunities to become a group leader were few and far in between. My dream was to lead my own research laboratory, and I thought the experience would be somewhat similar anywhere in the world: I work in bioinformatics, so I need few things besides good students and collaborators and computing facilities.

It was a difficult personal and professional decision. I had so many questions: will I be able to lead a research group? Will I get funding? Will I readjust to living in my home country? One of the main things that made me doubt my choice is that being a good scientist does not guarantee you will be a good mentor or lab head. This fact is not surprising, given our training is heavily focused on research and not on managing people and resources. During my postdoc I was the associate advisor of two $\mathrm{PhD}$ students, but my teaching experience was limited, and I had no experience in managing grants. My then partner now husband, who is also a scientist, had to decide whether to follow me without having secured a position himself. It was a time of great strain and uncertainty, compounded by the reverse culture shock I was experiencing and by my new, highly demanding position.

Becoming a principal investigator (PI) in Mexico has been both far more challenging and rewarding than I had imagined. Over the past few years, I have discovered I enjoy teaching and have an aptitude for teaching, mentoring students and writing grants. One of the big perks of working in Mexico is that most graduate-student stipends and the salaries of some researchers are paid for by federal government funding. This gives junior researchers the opportunity to get started quickly and be less reliant on external funding.

I spent my first year as PI applying for funding. I was glad to discover that, in addition to national funding, there are diverse funding sources from international foundations and organizations dedicated to junior researchers in developing countries. In 2016, I received the L'Oréal-UNESCO-AMC-CONACYT award for Women in Science national fellowship, and in 2018 I was selected as one of 15 L'Oréal-UNESCO International Rising Talents. This recognition has been instrumental for my career, by providing me with flexible funding to get my research projects started, raising my public profile among my peers and opening a series of opportunities I would otherwise not have access to. It has also been a great honour and responsibility to be a spokeswoman for STEM issues and to help inspire young Mexican women to consider a career in science.

Leading a lab is not for the faint hearted; I received my first substantial grant almost 3 years in and published our first research paper of work done entirely in Mexico only in April 2020. Managing people is rewarding, but also challenging and time consuming. However, seeing my students develop their skills and become independent scientists is incredibly fulfilling and, for many of them, truly life changing.

Last year I received a permanent position; I now have a research assistant and a team of postgraduate students and postdocs working on exciting projects and collaborations in Mexico and overseas. My husband found an academic position, and we recently expanded our family. I am deeply satisfied with my work.

The future of research in Mexico is uncertain. Scientists have now become indispensable for decisionmaking processes regarding the ongoing COVID-19 pandemic and of course for developing treatments for it. However, cuts to research funding continue and may have dire consequences for Mexican research. I hope the current crisis will encourage scientists to take a more prominent role in guiding public opinion and policy, in Mexico and across the globe.

Competing interests

The author declares no competing interests. 